\title{
Über den Einfluß von Substitution in den Komponenten binärer Lösungsgleichgewichte
}

\author{
(III. Mitteilung) \\ von
}

R. Kremann.

Nach experimentellen Versuchen der Herren E. Benesch, W. Decolle, P. Dolch, K. Kaas, F. Pilch und F. Scherenziss.

Aus dem chemischen Institut der Universität Graz.

(Mit 10 Textiguren.)

(Vorgelegt in der Sitzung am 9. Juli 1908.)

In einer früheren Mitteilung hatte ich gemeinsam mit O. Rodinis ${ }^{1}$ unter anderem gezeigt, daß die Fähigkeit von Naphthalin, mit den isomeren Dinitrobenzolen und Dinitrotoluolen Verbindungen zu bilden, außer von der Elektroaffinität der betreffenden Nitroverbindung, in erster Linie von der Stellung der beiden Nitrogruppen abhängt. Es hatte sich damals als Spezialfall einer bei mehreren analogen Fällen zu Recht bestehenden Regel ergeben, daß, während $m$ - und $p$-Dinitrobenzol mit Naphthalin Verbindungen in äquimolekularem Verhältnis liefern, o-Dinitrobenzol diese Fähigkeit nicht besitzt und von den damals untersuchten vier Dinitrotoluolen nur diejenigen, bei denen die beiden Nitrogruppen weder zueinander noch beide zugleich zur $\mathrm{CH}_{3}$-Gruppe in Orthostellung sich befinden, d. i. bei 1:2:4- und 1:3:5-Dinitrotoluol. Wir erk1ärten dieses unterschiedliche Verhalten durch sterische Valenzbehinderung, die bei diesen Orthostellungen auftritt.

Es war nun von einem gewissen Interesse, die Additionsfähigkeit der isomeren Dinitrobenzole und Dinitrotoluole gegen-

1 Monatshefte fuir Chemie, 27, 125 (1906). 
über anderen aromatischen Kohlenwasserstoffen, etwa Benzol und Phenanthren zu untersuchen und zu erfahren, ob die oben erwähnte Regel auch hier zu Recht besteht. Zumal wäre es von besonderem Interesse gewesen, für das System $m$-Dinitrobenzol-Naphthalin, bei welchem die äquimolekulare Verbindung dieser Stoffe im Schmelzfluß so weitgehend dissoziiert, daß der ihr zukommende Teil nahezu eine Horizontale darstellt - es ist dies ein von mir zum erstenmal beobachteter Fall ${ }^{1}$ - ein Analogon zu finden. Bei dem System $m$-Dinitrobenzol-Phenanthren, bei dem ziemlich analoge Temperaturverhältnisse obwalten, wie beim System $m$-Dinitrobenzol-Naphthalin, wäre von vornherein ein analoges Verhalten nicht ausgeschlossen.

Ich habe deshalb im vorigjährigen und diesjährigen physi . kalisch-chemischen Praktikum einige Herren veranlaßt; die diesbezüglichen Schmelzdiagramme aufzunehmen, über welche Versuche im experimentellen Teile unter Angabe des betreffenden Beobachters berichtet wird.

Als allgemeines Ergebnis ist zu verzeichnen, daß aus dem Verlauf der Schmelzkurven keinerlei Andeutungen dafür zu finden sind, daß irgendeines der drei isomeren Dinitrobenzole mit Benzol sowohl, als irgendeines der drei zur Untersuchung verwendeten isomeren Dinitrotoluole $(1,2,4,1,2,6$ und $1,3,4)$ als auch mit Phenanthren zu einer Verbindung zusammentreten. Von den drei isomeren Dinitrobenzolen gibt mit Phenanthren nicht nur das o-Dinitrobenzol, sondern auch das $m$-Dinitrobenzol keine Verbindung. Nur das $p$-Dinitrobenzol tritt mit Phenanthren, wie aus dem Verlauf des Schmelzdiagrammes und geeigneten Zeit-Ablühlungskurven zu ersehen ist, zu einer Verbindung zusammen, die sich aus 3 Molekülen Phenanthren und 1 Molekül $p$-Dinitrobenzol zusammensetzt. Thr Existenzbereich ist nur klein, es liegt zwischen 69 und 79 Molekülprozenten Phenanthren. Wir kommen also zum Schlusse, daß von den nunmehr untersuchten aromatischen Kohlenwasserstoffen mit einem, zwei und drei Benzolkernen der zweikernige, das Naphthalin, am fähigsten ist zur Bildung von Verbindungen. Während Naphthalin mit Ausnahme oben

1 Monatshefte für Chemie, 25, 1283 (1905). 
erwähnter Fälle sterischer Valenzbehinderung mit Dinitrobenzol und Dinitrotoluol Verbindungen liefert, geht Benzol wie Phenanthren diese Fähigkeit ab. Erst wenn wir die Elektroaffinität der erwähnten Dinitrokörper durch Einführung einer weiteren Nitrogruppe erhöhen, also Trinitrobenzol, beziehungsweise Trinitrotoluol verwenden, tritt die Additionsfähigkeit gegenüber Phenanthren wieder auf. In beiden Fällen ergibt sich aus dem Verlauf der Schmelzkurven der beiden Systeme PhenanthrenTrinitrobenzol und Phenanthren-Trinitrotoluol, daß Phenanthren sowohl mit Trinitrobenzol als auch mit Trinitrotoluol zu Verbindungen im äquimolekularen Verhältnis zusammentritt.

\section{Experimenteller Teil.}

Die Methode der Aufnahme der Schmelzdiagramme war die bekannte, von mir in der ersten Mitteilung ausführlich dargelegte.

\section{Lösungsgleichgewichte zwischen Benzol und den drei isomeren Dinitrobenzolen.}

DieVersuchsergebnisse geben beistehende Tabellen wieder.

Tabelle 1 (Beobachter: W. Decolle).

Lösungsgleichgewicht zwischen Benzol und $m$-Dinitrobenzol.

Zusatz von $m$-Dinitrobenzol zu Benzol.

\begin{tabular}{|c|c|c|c|}
\hline $\begin{array}{c}\text { Zusatz von } \\
\text { m-Dinitrobenzol }\end{array}$ & $\begin{array}{c}\text { Gewichtsprozente } \\
\text { Benzol }\end{array}$ & $\begin{array}{c}\text { Molekülprozente } \\
\text { Benzol }\end{array}$ & Schmelzpunkt \\
\hline \multicolumn{4}{|c|}{ a) Menge Benzol: $17 \cdot 043 \mathrm{~g}$} \\
\hline 0.000 & $100 \cdot 0$ & $100 \cdot 0$ & $5 \cdot 0$ \\
\hline $2 \cdot 330$ & $88 \cdot 0$ & $94 \cdot 5$ & $2 \cdot 2$ \\
\hline $3 \cdot 044$ & $84 \cdot 8$ & $92 \cdot 7$ & $1 \cdot 1$ \\
\hline $4 \cdot 514$ & $79 \cdot 1$ & $89 \cdot 1$ & 0.5 \\
\hline $5 \cdot 936$ & $74 \cdot 2$ & $86 \cdot 0$ & $4 \cdot 2$ \\
\hline $6 \cdot 663$ & $71 \cdot 9$ & $84 \cdot 6$ & $12 \cdot 0$ \\
\hline $7 \cdot 545$ & $69 \cdot 1$ & $82 \cdot 8$ & $17 \cdot 2$ \\
\hline $8 \cdot 647$ & $66 \cdot 3$ & $80 \cdot 9$ & $20 \cdot 0$ \\
\hline $9 \cdot 689$ & $63 \cdot 7$ & $79 \cdot 0$ & $22 \cdot 5$ \\
\hline $11 \cdot 224$ & $60: 3$ & $76 \cdot 5$ & $25 \cdot 4$ \\
\hline $13 \cdot 858$ & $55 \cdot 1$ & $72 \cdot 5$ & $30 \cdot 5$ \\
\hline
\end{tabular}




\begin{tabular}{|c|c|c|c|}
\hline $\begin{array}{c}\text { Zusatz von } \\
m \text {-Dinitrobenzol }\end{array}$ & $\begin{array}{c}\text { Gewichtsprozente } \\
\text { Benzol }\end{array}$ & $\begin{array}{c}\text { Molekülprozente } \\
\text { Benzol }\end{array}$ & Schmelzpunkt \\
\hline \multicolumn{4}{|c|}{ b) Menge Benzol: $14 \cdot 719 \mathrm{~g}$} \\
\hline $12 \cdot 590$ & $53 \cdot 9$ & $71 \cdot 5$ & $33 \cdot 0$ \\
\hline $15 \cdot 198$ & $49 \cdot 2$ & $67 \cdot 4$ & $.37 \cdot 5$ \\
\hline $18 \cdot 118$ & $44 \cdot 8$ & $63 \cdot 5$ & $42 \cdot 0$ \\
\hline $20 \cdot 003$ & $42 \cdot 4$ & $61 \cdot 2$ & $45 \cdot 0$ \\
\hline $22 \cdot 724$ & $39 \cdot 3$ & $58 \cdot 1$ & $47 \cdot 5$ \\
\hline $25 \cdot 609$ & $36 \cdot 5$ & $55 \cdot 3$ & $51 \cdot 0$ \\
\hline $29 \cdot 266$ & $33 \cdot 5$ & $52 \cdot 0$ & $53 \cdot 5$ \\
\hline $31 \cdot 597$ & $31 \cdot 8$ & $50 \cdot 1$ & $55 \cdot 0$ \\
\hline $34 \cdot 264$ & $30 \cdot 1$ & $48 \cdot 1$ & $57 \cdot 0$ \\
\hline \multicolumn{4}{|c|}{ c) Menge Benzol: $5 \cdot 271 \mathrm{~g}$} \\
\hline $15 \cdot 197$ & $25 \cdot 8$ & $43 \cdot 2$ & $59 \cdot 0$ \\
\hline $19 \cdot 446$ & $21 \cdot 3$ & $36 \cdot 8$ & $64 \cdot 5$ \\
\hline $23 \cdot 454$ & $18 \cdot 3$ & $32 \cdot 8$ & $68 \cdot 0$ \\
\hline $27 \cdot 728$ & $16 \cdot 0$ & $29 \cdot 1$ & $74 \cdot 5$ \\
\hline $32 \cdot 478$ & $13 \cdot 9$ & $26 \cdot 2$ & $75 \cdot 0$ \\
\hline $36 \cdot 108$ & $12 \cdot 7$ & $24 \cdot 3$ & $76 \cdot 0$ \\
\hline $39 \cdot 285$ & $11 \cdot 8$ & $22 \cdot 2$ & $77 \cdot 0$ \\
\hline $44 \cdot 815$ & $10 \cdot 5$ & $20 \cdot 5$ & $79 \cdot 0$ \\
\hline
\end{tabular}

Tabelle 2 (Beobachter: E. Benesch).

Lösungsgleichgewicht $z$ wischen Benzol und $o$-Dinitrobenzol.

Zusatz von o-Dinitrobenzol zu Benzol.

\begin{tabular}{|c|c|c|c|}
\hline $\begin{array}{c}\text { Zusatz von } \\
\text { Dinitrobenzol }\end{array}$ & $\begin{array}{c}\text { Gewichtsprozente } \\
\text { Benzol }\end{array}$ & $\begin{array}{c}\text { Molekülprozente } \\
\text { Benzol }\end{array}$ & Schmelzpunkt \\
\hline \multicolumn{3}{|c|}{ a) Menge Benzol: $4.733 g$} \\
\hline 0.000 & $100 \cdot 0$ & 100.0 & 5.0 \\
0.454 & 91.8 & 96.1 & 31.0 \\
0.811 & 85.5 & $93 \cdot 1$ & 39.0 \\
1.174 & 80.1 & 89.7 & 47.0 \\
1.508 & 75.8 & 86.8 & 53.0 \\
\end{tabular}


Einflub von Substitution etc.

\begin{tabular}{|c|c|c|c|}
\hline $\begin{array}{c}\text { Zusatz von } \\
\text { Dinitrobenzol }\end{array}$ & $\begin{array}{c}\text { Gewichtsprozente } \\
\text { Benzol }\end{array}$ & $\begin{array}{c}\text { Molekülprozente } \\
\text { Benzol }\end{array}$ & Schmelzpunkt \\
\hline $1 \cdot 951$ & $70 \cdot 8$ & $83 \cdot 9$ & $58 \cdot 0$ \\
$2 \cdot 711$ & $63 \cdot 6$ & $78 \cdot 9$ & $65 \cdot 0$ \\
$3 \cdot 295$ & $59 \cdot 0$ & $75 \cdot 6$ & $69 \cdot 0$ \\
$4 \cdot 373$ & $51 \cdot 9$ & $69 \cdot 9$ & $75 \cdot 0$ \\
$5 \cdot 591$ & $45 \cdot 8$ & $64 \cdot 5$ & $80 \cdot 0$ \\
$6 \cdot 813$ & $41 \cdot 0$ & $59 \cdot 8$ & $84 \cdot 0$ \\
$8 \cdot 263$ & $36 \cdot 4$ & $55 \cdot 2$ & $87 \cdot 0$ \\
$9 \cdot 971$ & $32 \cdot 2$ & $50 \cdot 8$ & $90 \cdot 0$ \\
$11 \cdot 370$ & $29 \cdot 4$ & $47 \cdot 3$ & $93 \cdot 0$ \\
$13 \cdot 347$ & $26 \cdot 2$ & $43 \cdot 5$ & $95 \cdot 5$ \\
\hline & b) Menge Benzol:6.644g & \\
\hline $0 \cdot 142$ & $97 \cdot 9$ & $98 \cdot 7$ & $4 \cdot 3$ \\
$0 \cdot 275$ & $94 \cdot 1$ & $97 \cdot 2$ & \\
& & &
\end{tabular}

Tabelle 3 (Beobachter: F. Pilch).

Lösungsgleichgewicht $z$ wischen $p$-Dinitrobenzol und Benzol

Zusatz von $p$-Dinitrobenzol zu Benzol.

\begin{tabular}{|c|c|c|c|}
\hline $\begin{array}{c}\text { Zusatz von } \\
\text { Dinitrobenzol }\end{array}$ & $\begin{array}{c}\text { Gewichtsprozente } \\
\text { Benzol }\end{array}$ & $\begin{array}{c}\text { Molekuilprozente } \\
\text { Benzol }\end{array}$ & Schmelzpunkt \\
\hline \multicolumn{3}{|c|}{ a) Menge Benzol: $9 \cdot 318 g$} \\
\hline $0 \cdot 000$ & $100 \cdot 0$ & $100 \cdot 0$ & $5 \cdot 2$ \\
0.111 & $98 \cdot 8$ & $99 \cdot 4$ & $4 \cdot 8$ \\
0.349 & $96 \cdot 4$ & $98 \cdot 3$ & $28 \cdot 01$ \\
0.498 & $94 \cdot 9$ & $97 \cdot 6$ & $40 \cdot 6$ \\
$0 \cdot 730$ & $92 \cdot 7$ & $96 \cdot 5$ & $54 \cdot 0$ \\
$0 \cdot 906$ & $91 \cdot 1$ & $95 \cdot 7$ & $61 \cdot 51$ \\
1 Lösungen angegebener Konzentration schieden Krystalle aus vom \\
Schmelzpunkt $172^{\circ}$ und erwiesen sich als reines $p$-Dinitrobenzol.
\end{tabular}




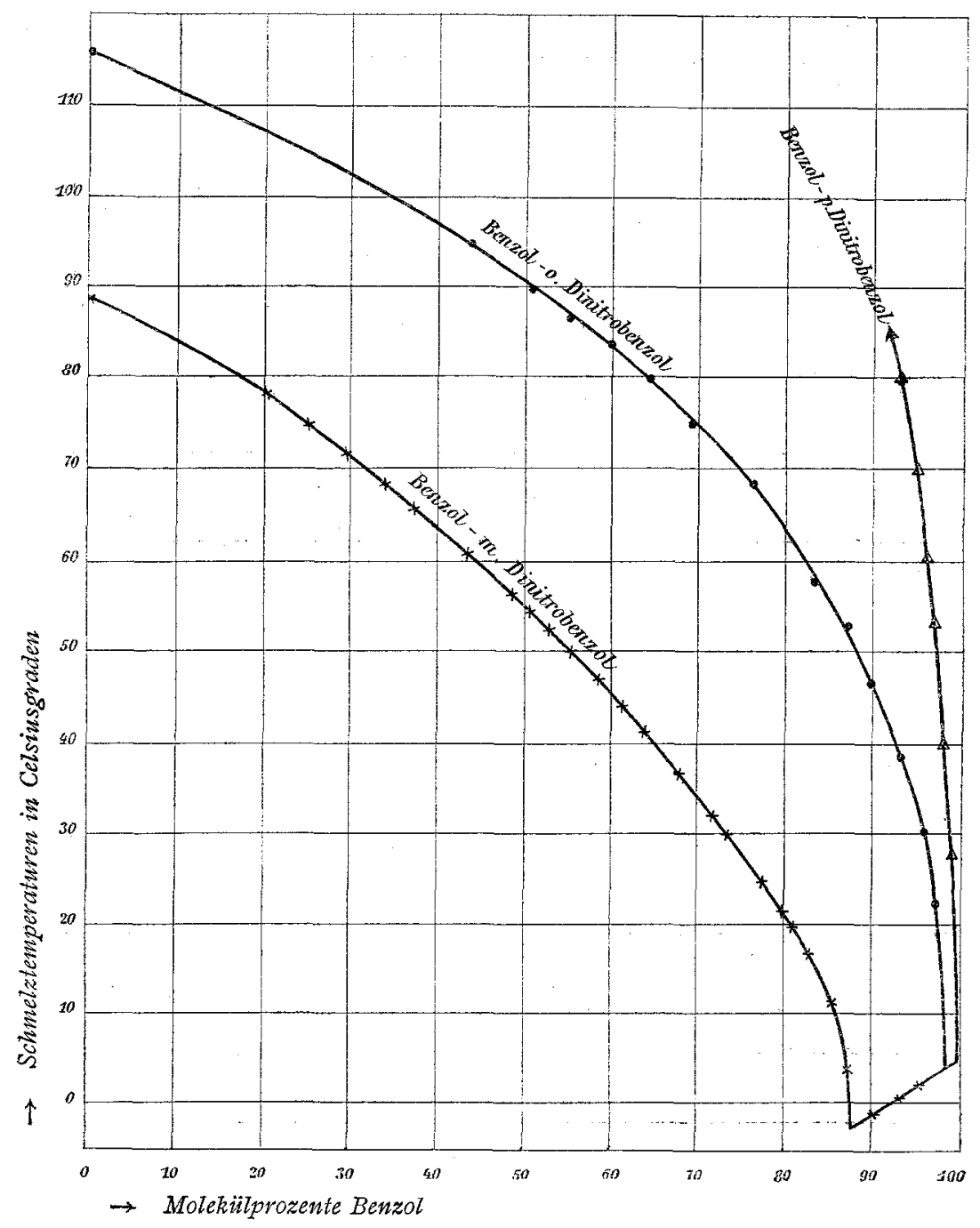

Fig. 1.

Lösungsgleichgewicht $z$ wischen Benzol und den drei isomeren Dinitrobenzolen. 


\begin{tabular}{|c|c|c|c|}
\hline $\begin{array}{l}\text { Zusatz von } \\
\text { Dinitrobenzol }\end{array}$ & $\begin{array}{c}\text { Gewichtsprozente } \\
\text { Benzol }\end{array}$ & $\begin{array}{c}\text { Molekülprozente } \\
\text { Benzol }\end{array}$ & Schmelzpunkt \\
\hline \multicolumn{4}{|c|}{ b) Menge Benzol: $10 \cdot 168 \mathrm{~g}$} \\
\hline $\begin{array}{l}1 \cdot 275 \\
1 \cdot 677\end{array}$ & $\begin{array}{l}88 \cdot 7 \\
85 \cdot 7\end{array}$ & $\begin{array}{l}94 \cdot 5 \\
92 \cdot 8\end{array}$ & $\begin{array}{l}70 \cdot 5 \\
78 \cdot 91\end{array}$ \\
\hline \multicolumn{4}{|c|}{$\begin{array}{l}1 \text { Bei } 80 \cdot 9^{\circ} \text { tritt Sieden des Benzols ein. Es ist also hier die } \\
\text { sogenannte } \$ \text { Kochgrenze" erreicht. Bei Zusammenbringen von Benzol und } \\
p \text {-Dinitrobenzol im Verhältnis von } 92 \cdot 0: 8 \cdot 0 \text { Molekülprozenten tritt eben } \\
\text { noch Lösung des } p \text {-Dinitrobenzols ein. Bei } 92 \cdot 0 \text { Molekülprozenten Benzol } \\
\text { beträgt der Schmelzpunkt also } 80 \cdot 9^{\circ} \text {. Dieser Punkt ist im Diagramm mit } \\
\text { bezeichnet. }\end{array}$} \\
\hline
\end{tabular}

Aus den die Versuchsergebnisse veranschaulichenden Diagrammen Fig. 1 ergibt sich, daß keines der drei Dinitrobenzole mit Benzol zu einer Verbindung zusammentritt. Die Löslichkeitslinien derselben in Benzol stellen stetige Kurven dar; die sich auch bei tieferen Temperaturen, etwa bei $10^{\circ}$, ausscheidenden Krystalle enthielten kein Benzol und erwiesen sich durch Bestimmung des Schmelzpunktes als reine Dinitrobenzole.

Bei einer Mischung von 70 Molekülprozenten Benzol und 30 Molekülprozenten $m$-Dinitrobenzol nahm ich eine Zeit-Abkühlungskurve auf. Ihr stetiger Verlauf zeigt, daß die geringen Unregelmäßigkeiten, die das Schmalzdiagramm $m$-Dinitrobenzol-Benzol etwa bei 80 Molekülprozenten Benzol aufTemperatur

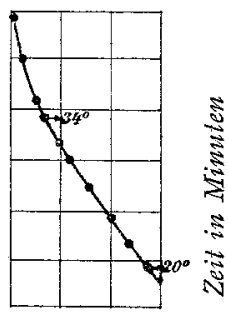

Fig. 2. weist, nicht durch das Auftreten einer Umwandlungserscheinung zurückzuführen ist, sondern seinen Grund in Beobachtungsfehlern hat.

Die Beobachtung des Thermometers während des Erstarrens einer Mischung obiger Zusammensetzung ergab:

$\begin{array}{cccc}\text { Zeit in Minuten } & \text { Temperatur } & \text { Zeit in Minuten } & \text { Temperatur } \\ 0 & 44 & 1 \cdot 5 & 34 \\ 0.5 & 40 & 2 \cdot 0 & 33 \\ 1.0 & 36 & 2 \cdot 5 & 31\end{array}$




$\begin{array}{cccc}\text { Zeit in Minuten } & \text { Temperatur } & \text { Zeit in Minuten } & \text { Temperatur } \\ 3 \cdot 0 & 30 & 6 \cdot 0 & 22 \\ 4 \cdot 0 & 27 & 7 \cdot 0 & 20 \\ 5 \cdot 0 & 24 & & \end{array}$

Die in Fig. 2 gegebene Zeit-Abkühlungskurve ergibt den Erstarrungspunkt, d. i. das Ausscheiden der ersten Krystalle bei $34^{\circ}$, was sich dem Verlauf der Schmelzkurve sehr gut einpaßt, bei tieferer Temperatur keine Andeutung für irgendeine Umwandlungserscheinung.

\section{Lösungsgleichgewichte zwischen Benzol und den isomeren Dinitrotoluolen: $1,2,4 ; 1,2,6 ; 1,3,4$.}

Die folgenden Tabellen geben die Versuchsergebnisse wieder, welche die Schmelzkurven der Fig. 3 veranschaulichen.

Tabelle 4 (Beobachter: E. Benesch).

Lösungsgleichgewicht zwischen Benzol und 1,2,4-Dinitrotoluol.

Zusatz von Dinitrotoluol zu Benzol.

\begin{tabular}{|c|c|c|c|}
\hline $\begin{array}{l}\text { Zusatz von } \\
\text { Dinitrotoluol }\end{array}$ & $\begin{array}{c}\text { Gewichtsprozente } \\
\text { Benzol }\end{array}$ & $\begin{array}{c}\text { Molekülprozente } \\
\text { Benzol }\end{array}$ & Schmelzpunkt \\
\hline \multicolumn{4}{|c|}{ a) Menge Benzol: $15 \cdot 688 g$} \\
\hline 0.000 & $100 \cdot 0$ & $100 \cdot 0$ & $5 \cdot 0$ \\
\hline $1 \cdot 511$ & $90 \cdot 4$ & $95 \cdot 4$ & $2 \cdot 5$ \\
\hline $2 \cdot 358$ & $86 \cdot 9$ & $93 \cdot 9$ & $1 \cdot 5$ \\
\hline $2 \cdot 846$ & $84 \cdot 6$ & $92 \cdot 6$ & $1 \cdot 0$ \\
\hline $3 \cdot 218$ & $83 \cdot 0$ & $92 \cdot 0$ & 0.5 \\
\hline $4 \cdot 289$ & $78 \cdot 5$ & $89 \cdot 0$ & -0.5 \\
\hline $5 \cdot 239$ & $75 \cdot 0$ & $87 \cdot 1$ & $+3 \cdot 5$ \\
\hline $6 \cdot 099$ & $72 \cdot 0$ & $85 \cdot 6$ & $+5 \cdot 5$ \\
\hline $7 \cdot 196$ & $68 \cdot 6$ & $83 \cdot 6$ & $+11 \cdot 0$ \\
\hline
\end{tabular}


Einflub von Substitution etc.

\begin{tabular}{|c|c|c|c|}
\hline $\begin{array}{l}\text { Zusatz von } \\
\text { Dinitrotoluol }\end{array}$ & $\begin{array}{c}\text { Gewichtsprozente } \\
\text { Benzol }\end{array}$ & $\begin{array}{l}\text { Moleküliprozente } \\
\text { Benzol }\end{array}$ & Schmelzpunkt \\
\hline $8 \cdot 485$ & $64 \cdot 9$ & $81 \cdot 1$ & $+15 \cdot 0$ \\
\hline $10 \cdot 022$ & $61 \cdot 0$ & $78 \cdot 6$ & $+18 \cdot 0$ \\
\hline $11 \cdot 346$ & $\therefore 58.0$ & $76 \cdot 4$ & $+22 \cdot 0$ \\
\hline $12 \cdot 422$ & $55 \cdot 8$ & $74 \cdot 7$ & +24.0 \\
\hline $13 \cdot 773$ & $53 \cdot 3$ & $72 \cdot 8$ & $+25 \cdot 0$ \\
\hline \multicolumn{4}{|c|}{ b) Menge Benzol: $17 \cdot 291 \mathrm{~g}$} \\
\hline $19 \cdot 457$ & $47 \cdot 0$ & $67 \cdot 5$ & $30 \cdot 0$ \\
\hline $20 \cdot 343$ & $45 \cdot 9$ & $66 \cdot 5$ & $30 \cdot 5$ \\
\hline $21 \cdot 302$ & $44 \cdot 7$ & $65 \cdot 4$ & $31 \cdot 5$ \\
\hline $23 \cdot 017$ & $42 \cdot 9$ & $63 \cdot 7$ & $33 \cdot 0$ \\
\hline $24 \cdot 806$ & $41 \cdot 1$ & $62 \cdot 0$ & $35 \cdot 0$ \\
\hline $26 \cdot 021$ & $39 \cdot 9$ & $60 \cdot 8$ & $35 \cdot 0$ \\
\hline $27 \cdot 666$ & $38 \cdot 5$ & $59 \cdot 4$ & $37 \cdot 0$ \\
\hline $29 \cdot 908$ & $36 \cdot 5$ & $57 \cdot 3$ & $38 \cdot 5$ \\
\hline $32 \cdot 378$ & $34 \cdot 7$ & $55 \cdot 4$ & $40 \cdot 0$ \\
\hline $34 \cdot 591$ & $33 \cdot 3$ & $53 \cdot 8$ & $41 \cdot 5$ \\
\hline \multicolumn{4}{|c|}{ c) Menge Benzol: $2 \cdot 460 g$} \\
\hline $5 \cdot 677$ & $30 \cdot 2$ & $50 \cdot 2$ & $46 \cdot 0$ \\
\hline $6 \cdot 643$ & $27 \cdot 0$ & $46 \cdot 6$ & $48 \cdot 0$ \\
\hline $7 \cdot 817$ & $24 \cdot 0$ & $42 \cdot 3$ & $50 \cdot 5$ \\
\hline $9 \cdot 679$ & $20 \cdot 3$ & $37 \cdot 3$ & $53 \cdot 0$ \\
\hline $12 \cdot 954$ & $16 \cdot 0$ & $31 \cdot 0$ & $57 \cdot 0$ \\
\hline $17 \cdot 416$ & $12 \cdot 4$ & $24 \cdot 6$ & $59 \cdot 0$ \\
\hline $23 \cdot 379$ & $9 \cdot 5$ & $19 \cdot 2$ & $62 \cdot 0$ \\
\hline $28 \cdot 804$ & $7 \cdot 9$ & $15 \cdot 6$ & $63 \cdot 0$ \\
\hline
\end{tabular}


Tabelle 5 (Beobachter F. Scherenziss).

Lösungsgleichgewicht zwischen Benzol und 1,2,6-Dinitrotoluol.

a) Zusatz von Benzol zu Dinitrotoluol.

Menge Dinitrotoluol: $10 \cdot 508 \mathrm{~g}$.

\begin{tabular}{|c|c|c|c|}
\hline $\begin{array}{l}\text { Zusatz von } \\
\text { Dinitrotoluol }\end{array}$ & $\begin{array}{c}\text { Gewichtsprozente } \\
\text { Benzol }\end{array}$ & $\begin{array}{c}\text { Moleküilprozente } \\
\text { Benzol }\end{array}$ & Schmelzpunkt \\
\hline 0.000 & 0.0 & 0.0 & . $\quad 65 \cdot 0$ \\
\hline 0.151 & $1 \cdot 4$ & $2 \cdot 8$ & $63 \cdot 2$ \\
\hline $1 \cdot 265$ & $10 \cdot 7$ & $21 \cdot 2$ & $53 \cdot 3$ \\
\hline $1 \cdot 726$ & $14 \cdot 1$ & $27 \cdot 8$ & $49 \cdot 4$ \\
\hline $2 \cdot 155$ & $17 \cdot 0$ & $32 \cdot 5$ & $46 \cdot 5$ \\
\hline $2 \cdot 657$ & $20 \cdot 5$ & $37 \cdot 5$ & $43 \cdot 5$ \\
\hline 3.681 & $26 \cdot 1$ & $45 \cdot 0$ & $38 \cdot 0$ \\
\hline $4 \cdot 297$ & $29 \cdot 2$ & $49 \cdot 0$ & $36 \cdot 4$ \\
\hline $5 \cdot 596$ & $34 \cdot 7$ & $55 \cdot 3$ & $33 \cdot 0$ \\
\hline $6 \cdot 828$ & $39 \cdot 4$ & $60 \cdot 3$ & $30 \cdot 0$ \\
\hline $9 \cdot 071$ & $46 \cdot 3$ & $66 \cdot 8$ & $25 \cdot 0$ \\
\hline $10 \cdot 408$ & $49 \cdot 8$ & $69 \cdot 8$ & $22 \cdot 6$ \\
\hline
\end{tabular}

b) Zusatz von Dinitrotoluol zu Benzol.

Menge Benzol: $8 \cdot 511 \mathrm{~g}$.

\begin{tabular}{|c|c|c|c|}
\hline $\begin{array}{c}\text { Zusatz von } \\
\text { Dinitrotoluol }\end{array}$ & $\begin{array}{c}\text { Gewichtsprozente } \\
\text { Benzol }\end{array}$ & $\begin{array}{c}\text { Molekülprozente } \\
\text { Benzol }\end{array}$ & Schmelzpunkt \\
\hline \hline $0 \cdot 000$ & $100 \cdot 0$ & $100 \cdot 0$ & $5 \cdot 3$ \\
0.789 & 91.5 & $96 \cdot 2$ & $2 \cdot 8$ \\
1.837 & $82 \cdot 3$ & $91 \cdot 3$ & $0 \cdot 1$ \\
$2 \cdot 787$ & $75 \cdot 3$ & $87 \cdot 3$ & $3 \cdot 8$ \\
3.817 & $70 \cdot 4$ & $84 \cdot 9$ & $9 \cdot 0$ \\
$4 \cdot 924$ & $63 \cdot 3$ & $80 \cdot 2$ & $13 \cdot 0$ \\
$6 \cdot 121$ & $58 \cdot 2$ & $76 \cdot 6$ & $17 \cdot 0$ \\
$7 \cdot 323$ & $53 \cdot 8$ & $73 \cdot 0$ & $19 \cdot 5$ \\
8.375 & $50 \cdot 4$ & $70 \cdot 3$ & $21 \cdot 8$ \\
& & &
\end{tabular}


Tabelle 6 (Beobachter F. Pilch).

Lösungsgleichgewicht $z$ wischen Dinitrotoluol $(1,3,4)$ und Benzol.

a) Zusatz von Dinitrotoluol zu Benzol.

\begin{tabular}{|c|c|c|c|}
\hline $\begin{array}{l}\text { Zusatz von } \\
\text { Dinitrotoluol }\end{array}$ & $\begin{array}{c}\text { Gewichtsprozente } \\
\text { Benzol }\end{array}$ & $\begin{array}{c}\text { Molekülprozente } \\
\text { Benzol }\end{array}$ & Schmelzpunkt \\
\hline \multicolumn{4}{|c|}{ Menge Benzol: $9 \cdot 862 g$} \\
\hline $\begin{array}{r}0 \cdot 000 \\
0 \cdot 542 \\
0 \cdot 890 \\
1 \cdot 397 \\
1 \cdot 996 \\
3 \cdot 109 \\
4 \cdot 579 \\
5 \cdot 573 \\
7 \cdot 904 \\
8 \cdot 654 \\
10 \cdot 328\end{array}$ & $\begin{array}{r}100 \cdot 0 \\
94 \cdot 8 \\
91 \cdot 8 \\
87 \cdot 7 \\
83 \cdot 3 \\
76 \cdot 2 \\
68 \cdot 5 \\
63 \cdot 3 \\
55 \cdot 8 \\
54 \cdot 6 \\
49 \cdot 1\end{array}$ & $\begin{array}{r}100 \cdot 0 \\
97 \cdot 7 \\
96 \cdot 3 \\
94 \cdot 3 \\
92 \cdot 1 \\
88 \cdot 0 \\
83 \cdot 8 \\
80 \cdot 1 \\
74 \cdot 6 \\
73 \cdot 7 \\
69 \cdot 4\end{array}$ & $\begin{array}{r}5.1 \\
4.0 \\
3.4 \\
2.7 \\
2.0 \\
0.2 \\
-2.0 \\
-3.2 \\
-2.0 \\
-0.6 \\
--6.6\end{array}$ \\
\hline \multicolumn{4}{|c|}{ Menge Benzol: $3 \cdot 323 \mathrm{~g}$} \\
\hline $2 \cdot 520$ & $57 \cdot 0$ & $75 \cdot 5$ & $-5 \cdot 01$ \\
\hline \multicolumn{4}{|c|}{$\begin{array}{l}1 \text { Durch Aufnahme einer Zeit-Abkühlungskurve wurde der eutektische } \\
\text { at bei }-5 \cdot 0 \text { festgelegt, was im Diagramm Fig. } 3 \text { durch die eutektische } \\
\text { Zontale } e e_{1} \text { angedeutet ist. }\end{array}$} \\
\hline
\end{tabular}

b) Zusatz von Benzol zu Dinitrotoluol.

Menge Dinitrotoluol : $9 \cdot 499 \mathrm{~g}$.

\begin{tabular}{|c|c|c|c|}
\hline $\begin{array}{l}\text { Zusatz von } \\
\text { Benzol }\end{array}$ & $\begin{array}{c}\text { Gewichtsprozente } \\
\text { Benzol }\end{array}$ & $\begin{array}{c}\text { Molekülprozente } \\
\text { Benzol }\end{array}$ & Schmelzpunkt \\
\hline $\begin{array}{l}0 \cdot 000 \\
1 \cdot 308 \\
1 \cdot 663 \\
2 \cdot 383 \\
3 \cdot 261 \\
3 \cdot 993 \\
4 \cdot 813 \\
5 \cdot 639 \\
7 \cdot 739\end{array}$ & $\begin{array}{r}0 \cdot 0 \\
12 \cdot 1 \\
14 \cdot 9 \\
19 \cdot 6 \\
25 \cdot 7 \\
29 \cdot 6 \\
33 \cdot 6 \\
37 \cdot 2 \\
44 \cdot 9\end{array}$ & $\begin{array}{r}0 \cdot 0 \\
24 \cdot 3 \\
29 \cdot 5 \\
36 \cdot 3 \\
44 \cdot 6 \\
49 \cdot 5 \\
54 \cdot 2 \\
58 \cdot 1 \\
65 \cdot 5\end{array}$ & $\begin{array}{l}59 \cdot 0 \\
46 \cdot 0 \\
42 \cdot 5 \\
38 \cdot 2 \\
32 \cdot 5 \\
28 \cdot 6 \\
25 \cdot 0 \\
20 \cdot 5 \\
11 \cdot 8\end{array}$ \\
\hline
\end{tabular}




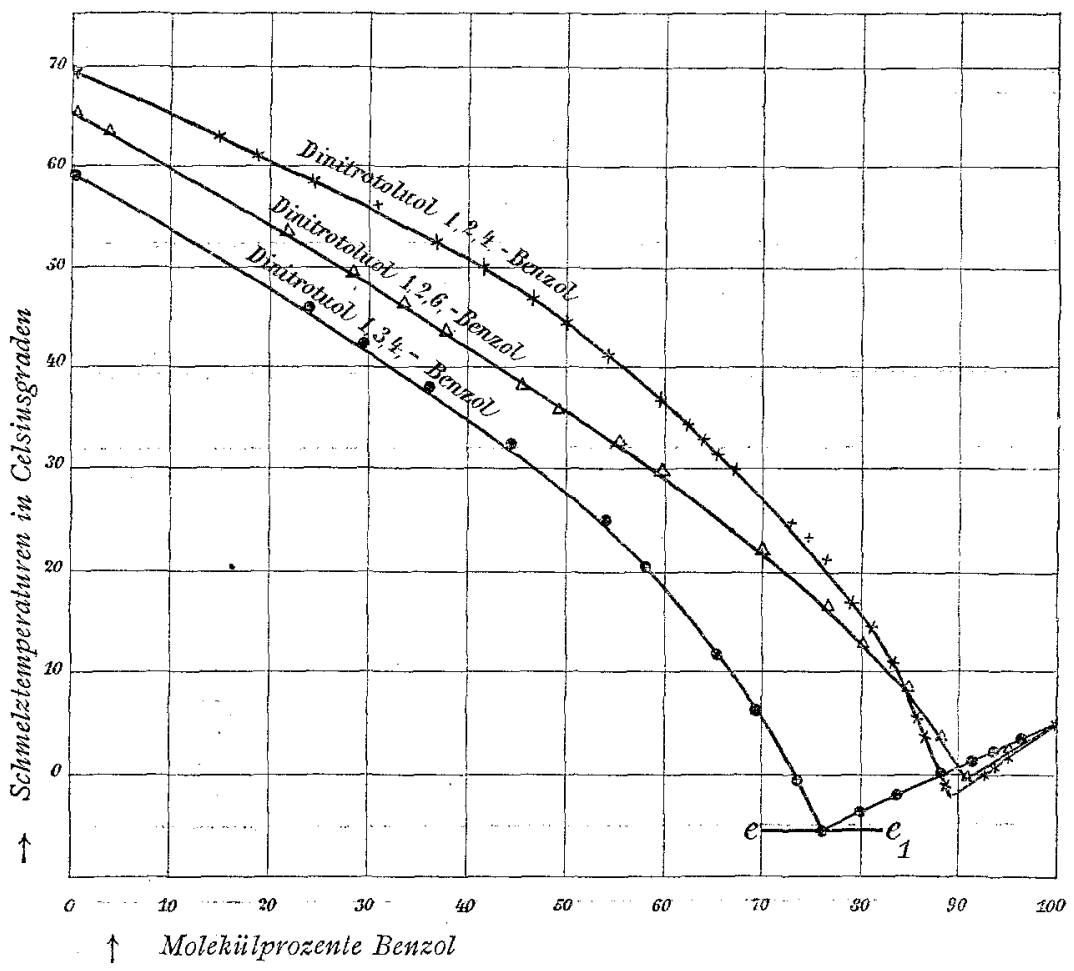

Fig. 3.

Lösungsgleichgewichte zwischen Dinitrotoluolen und Benzol.

Man sieht, daß auch hier keinerlei Andeutung sich für die Existenz von Verbindungen vorfindet. Die Löslichkeitskurven

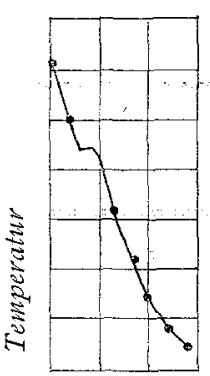

Fig. 4.

Zeit in Minuten nitrotoluol gab bei der langsamen Abkühlung unter stetem der Dinitrotoluole in Benzol verlaufen stetig. Die Lage der eutektischen Punkte ist ohneweiters aus den Diagrammen ersichilich.

Bei der Kurve Benzol-1,2,4-Dinitrotoluol finden sich bei zirka 75 Molekülprozent Benzol einige Unregelmäßigkeiten. Daß dies jedoch nur auf Beobachtungsfehler zurückzuführen ist und jede Umwandlungserscheinung fehlt, beweist das aufgenommene Zeit-Abkühlungsdiagramm. Eine Mischung von 60 Molekülprozenten Benzol und 40 Molekülprozenten Di- 
Rühren der Reihe nach folgende Temperaturen bei Ablesung: des Thermometers wälarend je einer Minute:

$$
46,40,37,31,26,22,19,17 \cdot 5^{\circ} \text {. }
$$

Die graphische Darstellung der Zeit-Abkühlungskurve in Fig. 4 zeigt, daß nur ein kurzes Anhalten des Thermometers bei dem Auftreten der ersten Krystalle, d. i. bei $37^{\circ}$, zu beobachten war. Unterhalb dieser Temperatur ist bis $17.5^{\circ}$ eine Umwandlungserscheinung nicht $\mathrm{zu}$ beobachten gewesen.

Bemerken möchte ich nur, daß die Löslichkeitskurven von 1,2,4-Dinitrotoluol und 1,2,6-Dinitrotoluol bei $9^{\circ}$ sich schneiden. Wir haben es also unterhalb $9^{\circ}$ mit einer Abweichung der Regel von Thomson und Carnalle $\mathrm{y}^{1}$ zu tun.

3. Lösungsgleichgewichte zwischen Phenanthren und den drei isomeren Dinitrobenzolen.

Die Versuchsergebnisse sind in den folgenden drei Tabellen wiedergegeben und in Fig. 5 graphisch dargestellt.

Tabelle 7 (Beobachter: W. Decolle).

Lösungsgleichgewicht zwischen $o$-Dinitrobenzol und Phenanthren.

a) Zusatz von Phenanthren zu Dinitrobenzol.

Menge Dinitrobenzol: $5 \cdot 829 \mathrm{~g}$.

\begin{tabular}{|c|c|c|c|}
\hline $\begin{array}{l}\text { Zusatz von } \\
\text { Phenanthren }\end{array}$ & $\begin{array}{c}\text { Gewichtsprozente } \\
\text { Phenanthren }\end{array}$ & $\begin{array}{l}\text { Molekülprozente } \\
\text { Phenanthren }\end{array}$ & Schmelzpunkt \\
\hline 0.000 & 0.0 & $0 \cdot 0$ & $116 \cdot 0$ \\
\hline $1 \cdot 27.3$ & $17 \cdot 9$ & $17 \cdot 1$ & $107 \cdot 1$ \\
\hline $1 \cdot 862$ & $24 \cdot 2$ & $23 \cdot 1$ & $104 \cdot 0$ \\
\hline $3 \cdot 052$ & $34 \cdot 4$ & $33 \cdot 1$ & $98 \cdot 0$ \\
\hline $4 \cdot 606$ & $44 \cdot 2$ & $42 \cdot 7$ & $91 \cdot 5$ \\
\hline $6 \cdot 759$ & $53 \cdot 7$ & $51 \cdot 2$ & $83 \cdot 5$ \\
\hline $8 \cdot 187$ & $58 \cdot 4$ & $56 \cdot 9$ & $78 \cdot 0$ \\
\hline
\end{tabular}

1 Journ. Chem. Soc., 53, 782 (1888), und Monatshefte für Chemie, 28,7 (1907). 
b) Zusatz von Dinitrobenzol zu Phenanthren.

Menge Phenanthren: $5 \cdot 294 \mathrm{~g}$.

\begin{tabular}{|c|c|c|c|}
\hline $\begin{array}{c}\text { Zusatz von } \\
\text { Dinitrobenzol }\end{array}$ & $\begin{array}{c}\text { Gewichtsprozente } \\
\text { Phenanthren }\end{array}$ & $\begin{array}{c}\text { Molekülprozente } \\
\text { Phenanthren }\end{array}$ & Schmelzpunkt \\
\hline 0.000 & $100 \cdot 0$ & $100 \cdot 0$ & $103 \cdot 0$ \\
0.591 & $89 \cdot 9$ & $89 \cdot 3$ & $96 \cdot 5$ \\
1.191 & 81.6 & $80 \cdot 7$ & $90 \cdot 2$ \\
2.520 & 57.8 & $66 \cdot 5$ & $79 \cdot 0$ \\
3.805 & 58.2 & $56 \cdot 7$ & $79 \cdot 5$ \\
& & & \\
\hline
\end{tabular}

Tabelle 8 (Beobachter: K. Kaas).

Lösungsgleichgewicht $z$ wischen $m$-Dinitrobenzol und Phenanthren.

a) Zusatz von $m$-Dinitrobenzol zu Phenanthren.

Menge Phenanthren: $8.787^{\circ}$.

\begin{tabular}{|c|c|c|c|}
\hline $\begin{array}{c}\text { Zusatz von } \\
\text { Dinitrobenzol }\end{array}$ & $\begin{array}{c}\text { Gewichtsprozente } \\
\text { Phenanthren }\end{array}$ & $\begin{array}{l}\text { Molekiilprozente } \\
\text { Phenanthren }\end{array}$ & Schmelzpunkt \\
\hline 0.000 & $100 \cdot 0$ & $100 \cdot 0$ & $103 \cdot 5$ \\
\hline $0 \cdot 420$ & $95 \cdot 4$ & $95 \cdot 2$ & $100 \cdot 0$ \\
\hline 0.939 & $90 \cdot 2$ & $89 \cdot 7$ & $96 \cdot 0$ \\
\hline $1 \cdot 791$ & $82 \cdot 9$ & $82 \cdot 1$ & $89 \cdot 0$ \\
\hline $2 \cdot 545$ & $77 \cdot 4$ & $76 \cdot 3$ & $84 \cdot 5$ \\
\hline $3 \cdot 622$ & $70 \cdot 6$ & $69 \cdot 3$ & $77 \cdot 0$ \\
\hline $4 \cdot 774$ & $64 \cdot 5$ & $63 \cdot 2$ & $70 \cdot 0$ \\
\hline $5 \cdot 957$ & $59 \cdot 4$ & $57 \cdot 9$ & $64 \cdot \overline{5}$ \\
\hline $7 \cdot 199$ & $54 \cdot 7$ & $53 \cdot 2$ & $58 \cdot 0$ \\
\hline $8 \cdot 194$ & $51 \cdot 5$ & $50 \cdot 0$ & $51 \cdot 0$ \\
\hline $9 \cdot 050$ & $49 \cdot 0$ & $47 \cdot 5$ & $48 \cdot 5$ \\
\hline $10 \cdot 440$ & $45 \cdot 4$ & $44 \cdot 0$ & $53 \cdot 0$ \\
\hline
\end{tabular}


b) Zusatz von Phenanthren zu $m$-Dinitrobenzol.

Menge $m$-Dinitrobenzol: $5 \cdot 086 \mathrm{~g}$.

\begin{tabular}{|c|c|c|c|}
\hline $\begin{array}{c}\text { Zusatz von } \\
\text { Phenanthren }\end{array}$ & $\begin{array}{c}\text { Gewichtsprozente } \\
\text { Phenanthren }\end{array}$ & $\begin{array}{c}\text { Molekülprozente } \\
\text { Phenanthren }\end{array}$ & Schmelzpunkt \\
\hline $0 \cdot 000$ & $0 \cdot 0$ & $0 \cdot 0$ & $89 \cdot 5$ \\
$0 \cdot 253$ & $4 \cdot 7$ & $4 \cdot 5$ & $87 \cdot 0$ \\
$0 \cdot 596$ & $10 \cdot 5$ & $10 \cdot 0$ & $83 \cdot 0$ \\
$0 \cdot 975$ & $16 \cdot 1$ & $15 \cdot 3$ & $80 \cdot 0$ \\
$1 \cdot 299$ & $20 \cdot 3$ & $19 \cdot 4$ & $76 \cdot 5$ \\
$1 \cdot 773$ & $25 \cdot 9$ & $24 \cdot 7$ & $72 \cdot 0$ \\
$2 \cdot 243$ & $30 \cdot 6$ & $29 \cdot 4$ & $69 \cdot 0$ \\
$2 \cdot 580$ & $33 \cdot 7$ & $32 \cdot 4$ & $65 \cdot 5$ \\
$3 \cdot 056$ & $37 \cdot 5$ & $36 \cdot 2$ & $62 \cdot 5$ \\
$3 \cdot 576$ & $41 \cdot 3$ & $39 \cdot 9$ & $58 \cdot 0$ \\
$4 \cdot 234$ & $45 \cdot 4$ & $44 \cdot 0$ & $53 \cdot 0$ \\
$4 \cdot 8419$ & $48 \cdot 8$ & $47 \cdot 3$ & $48 \cdot 5$ \\
$5 \cdot 425$ & $51 \cdot 6$ & $50 \cdot 2$ & - \\
$5 \cdot 777$ & $53 \cdot 2$ & $51 \cdot 8$ & $53 \cdot 0$ \\
$6 \cdot 154$ & $54 \cdot 8$ & $53 \cdot 3$ & $55 \cdot 5$ \\
$6 \cdot 445$ & $55 \cdot 9$ & $54 \cdot 5$ & $57 \cdot 0$ \\
$6 \cdot 716$ & $56 \cdot 9$ & $55 \cdot 5$ & $60 \cdot 0$ \\
$7 \cdot 089$ & $58 \cdot 3$ & $56 \cdot 8$ & $62 \cdot 0$ \\
$7 \cdot 695$ & $60 \cdot 2$ & $58 \cdot 8$ & $65 \cdot 0$ \\
& & & \\
& & & \\
\hline
\end{tabular}

Tabelle 9 (Beobachter: F. Pilch).

Lösungsgleichgewicht zwischen $p$-Dinitrobenzol und Phenanthren.

a) Zusatz von $p$-Dinitrobenzol zu Phenanthren.

\begin{tabular}{|c|c|c|c|}
\hline $\begin{array}{l}\text { Zusatz von } \\
\text { Dinitrobenzol }\end{array}$ & $\begin{array}{c}\text { Gewichtsprozente } \\
\text { Phenanthren }\end{array}$ & $\begin{array}{l}\text { Molekülprozente } \\
\text { Phenanthren }\end{array}$ & Schmelzpunkt \\
\hline \multicolumn{4}{|c|}{ a) Menge Phenanthren: $11 \cdot 024 \mathrm{~g}$} \\
\hline $0 \cdot 000$ & $100 \cdot 0$ & $100 \cdot 0$ & $103 \cdot 0$ \\
\hline $0 \cdot 649$ & $94 \cdot 4$ & $94 \cdot 1$ & $98 \cdot 5$ \\
\hline $1 \cdot 277$ & $89 \cdot 7$ & $89 \cdot 1$ & $94 \cdot 0$ \\
\hline $1 \cdot 914$ & $85 \cdot 2$ & $84 \cdot 4$ & $89 \cdot 5$ \\
\hline $3 \cdot 026$ & $78 \cdot 5$ & $77 \cdot 5$ & $81 \cdot 0$ \\
\hline $4 \cdot 138$ & $72 \cdot 7$ & $71 \cdot 5$ & $80 \cdot 3$ \\
\hline
\end{tabular}




\begin{tabular}{|c|c|c|c|}
\hline $\begin{array}{l}\text { Zusatz von } \\
\text { Dinitrobenzol }\end{array}$ & $\begin{array}{c}\text { Gewichtsprozente } \\
\text { Phenanthren }\end{array}$ & $\begin{array}{l}\text { Molekülprozente } \\
\text { Phenanthren }\end{array}$ & Schmelzpunkt \\
\hline \multicolumn{4}{|c|}{ B) Menge Phenanthren: $4.208 \mathrm{~g}$} \\
\hline $1 \cdot 731$ & $70 \cdot 9$ & $69 \cdot 6$ & $81 \cdot 0$ \\
\hline $2 \cdot 498$ & $62 \cdot 8$ & $61 \cdot 4$ & $104 \cdot 0$ \\
\hline $2 \cdot 986$ & $58 \cdot 5$ & $57 \cdot 1$ & $113 \cdot 0$ \\
\hline $3 \cdot 402$ & $55 \cdot 3$ & $53 \cdot 9$ & $119 \cdot 0$ \\
\hline $3 \cdot 789$ & $52 \cdot 6$ & $51 \cdot 2$ & $124 \cdot 0$ \\
\hline $4 \cdot 284$ & $49 \cdot 6$ & $48 \cdot 1$ & $129 \cdot 5$ \\
\hline \multicolumn{4}{|c|}{ ү) Menge Phenanthren: $5 \cdot 747 \mathrm{~g}$} \\
\hline $1 \cdot 758$ & $76 \cdot 6$ & $75 \cdot 5$ & $81 \cdot 0$ \\
\hline $1 \cdot 890$ & $75 \cdot 3$ & $76 \cdot 2$ & $80 \cdot 5$ \\
\hline $5 \cdot 263$ & $71 \cdot 8$ & $70 \cdot 6$ & $79 \cdot 0$ \\
\hline $2 \cdot 462$ & $70 \cdot 0$ & $68 \cdot 8$ & $84 \cdot 0$ \\
\hline
\end{tabular}

b) Zusatz von Phenanthren zu Dinitrobenzol.

Menge $p$-Dinitrobenzol : $11 \cdot 184 g$.

\begin{tabular}{|c|c|c|c|}
\hline $\begin{array}{l}\text { Zusatz von } \\
\text { Phenanthren }\end{array}$ & $\begin{array}{c}\text { Gewichtsprozente } \\
\text { Phenanthren }\end{array}$ & $\begin{array}{l}\text { Molekülprozente } \\
\text { Phenanthren }\end{array}$ & Schmelzpunkt \\
\hline 0.000 & 0.0 & 0.0 & $172 \cdot 0$ \\
\hline 0.513 & $4 \cdot 4$ & $4 \cdot 1$ & $170 \cdot 3$ \\
\hline $1 \cdot 042$ & $8 \cdot 5$ & $8 \cdot 0$ & $168 \cdot 0$ \\
\hline $1 \cdot 749$ & $13 \cdot 5$ & $12 \cdot 8$ & $165 \cdot 0$ \\
\hline $2 \cdot 829$ & $20 \cdot 1$ & $19 \cdot 2$ & $160 \cdot 0$ \\
\hline $3 \cdot 901$ & $25 \cdot 9$ & $24 \cdot 8$ & $155 \cdot 5$ \\
\hline $5 \cdot 221$ & $31 \cdot 8$ & $30 \cdot 6$ & $150 \cdot 0$ \\
\hline $6 \cdot 348$ & $36 \cdot 2$ & $34 \cdot 9$ & $145 \cdot 5$ \\
\hline $7 \cdot 373$ & $39 \cdot 7$ & $38 \cdot 4$ & $141 \cdot 5$ \\
\hline $8 \cdot 438$ & $43 \cdot 0$ & $41 \cdot 6$ & $137 \cdot 6$ \\
\hline
\end{tabular}




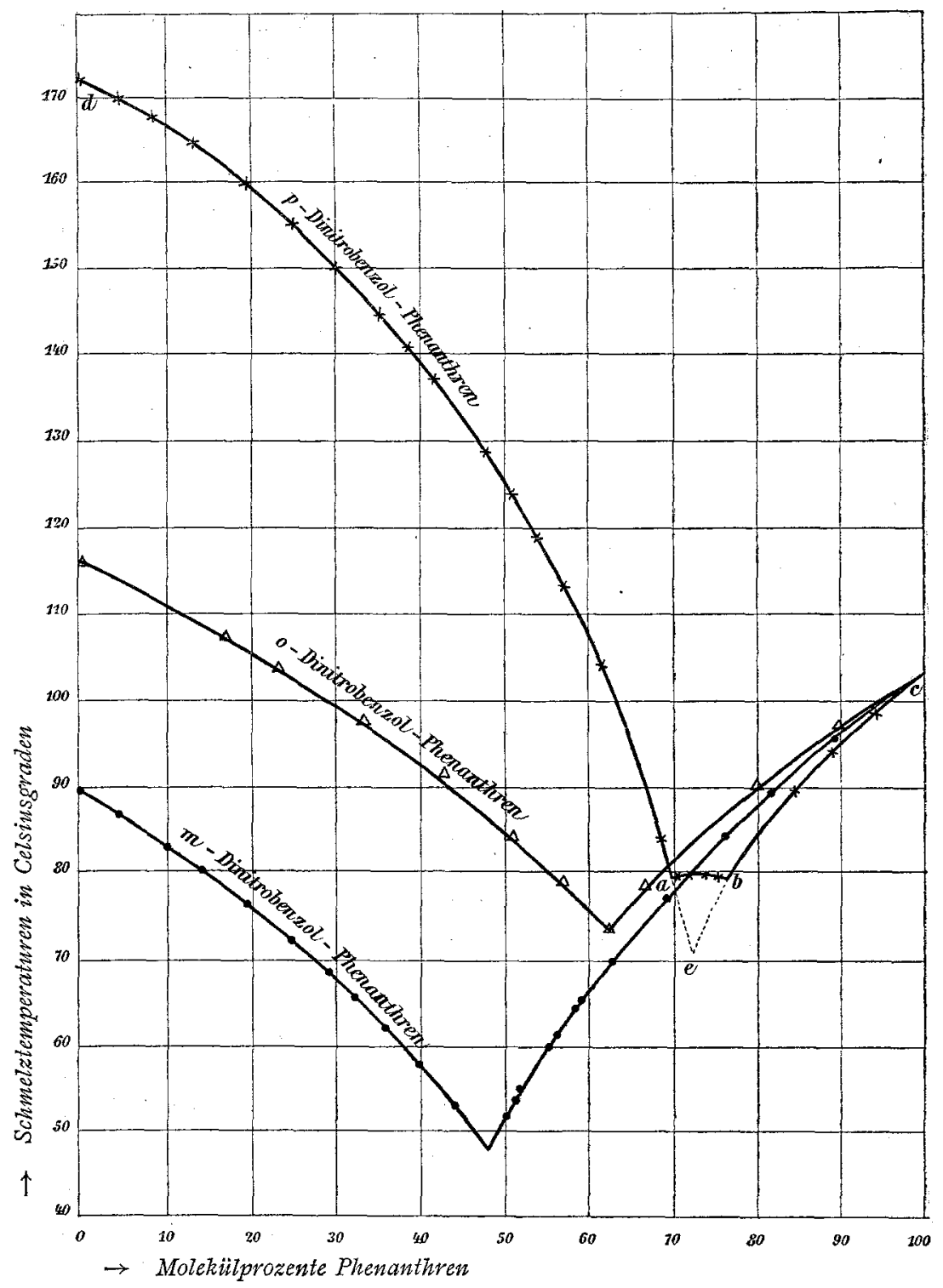

Fig. 5.

Lösungsgleichgewichte der drei isomeren Dinitrobenzole und Phenanthren.

Man sieht ohneweiters, daß weder o-Dinitrobenzol noch $m$-Dinitrobenzol mit Phenanthren zu einer Verbindung zusammentritt. Die beiden diesbezüglichen Schmelzdiagramme 
stellen je zwei stetig verlaufende Kurven dar, die Löslichkeitslinien von Phenanthren einerseits, der beiden Dinitrotoluole andrerseits. Der eutektische Punkt beider Systeme ergibt sich ohneweiters aus den Diagrammen.

Bei dem System $p$-Dinitrobenzol-Phenanthren findet sich ein Stück $a b$ zwischen 69 und 77 Molekülprozent Phenanthren, das weder der Löslichkeitskurve von Phenanthren $b c$ noch der von $p$.Dinitrobenzol angehört. Es dürfte sich hier um das Auftreten einer Verbindung handeln, die nach der Lage im Schmelzdiagramm die Zusammensetzung von 3 Molekülen Phenanthren und 1 Molekül Dinitrobenzol haben dürfte. Ich habe deshalb

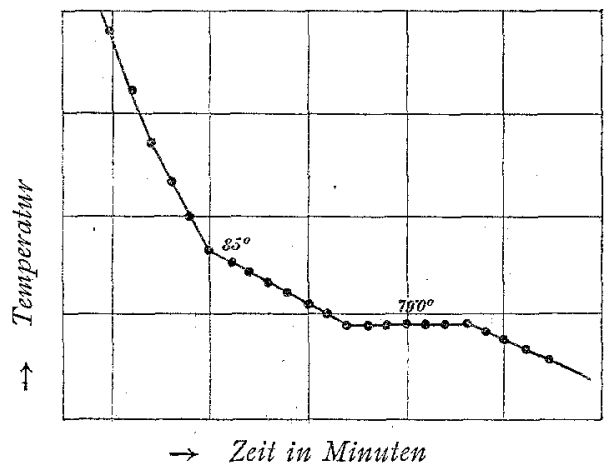

Fig. 6.

zur Festlegung der beiden eutektischen Punkte $a$ und $b$ sowie des Schmelzpunktes der Verbindung $f$ Zeit-Abkühlungskurven aufgenommen. Denn wenn keine Verbindung zwischen Phenanthren und $p$-Dinitrobenzol existierte, so würde sich nur ein eutektischer Punkt, und zwar im Schnittpunkt der beiden Löslichkeitslinien $e$, d. i. bei $71^{\circ}$ realisieren lassen müssen.

Eine Mischung, die 80 Molekülprozent Phenanthren enthielt, also auf der Löslichkeitskurve von Phenanthren liegend, reines Phenanthren ausscheidet, wurde langsam unter stetem Rühren erstarren gelassen und die Temperatur der Schmelze während jeder Minute abgelesen. Es wurden der Reihe nach folgende Temperaturwerte erhalten:

$108,102,97,93,90,86,85,84,83,82,81,80,79,79,79,79,79$, $79,79,78 \cdot 5,78,77,76$. (Bei diesen letzten Bestimmungen war bereits alles fest.) 
Man sieht aus der Zeit-Abkühlungskurve der Fig. 6, daß die erste Krystallausscheidung bei $85^{\circ}$ eintritt, wie sich auch aus dem Verlauf der Löslichkeitskurve Phenanthren $b c$ ableiten läßt, der eutektische Punkt $b$ bei $79^{\circ}$ liegt und nicht tiefer bei $71^{\circ}$.

Eine Mischung von 74 Molekülprozent gab während der Erstarrung folgende Temperaturwerte:

$106,101,97,92 \cdot 5,88 \cdot 5,86,84,82,81,81,81,80 \cdot 5,80,80,79 \cdot 5$, $79 \cdot 5.79 \cdot 5,79 \cdot 5,79,78 \cdot 5,78,77 \cdot 5,77,76 \cdot 5$.

Wir befinden uns bei der Mischung dieser Zusammensetzung auf der Löslichkeitskurve $a b$ der Verbindung. Wir sehen aus dem Verlauf der Zeit-Abkühlungskurve Fig. 7, daß bei $81^{\circ}$ ein Anhalten des Thermometers stattfindet, nach einiger tritt Sinken ein, bis bei $79 \cdot 5^{\circ}$ wieder ein neuerliches Anhalten der Temperatur zu beobachten ist. Dann tritt unter gleichzeitigem vollkommenen Festwerden der Masse stetes Sinken ein.

Es scheidet sich eben

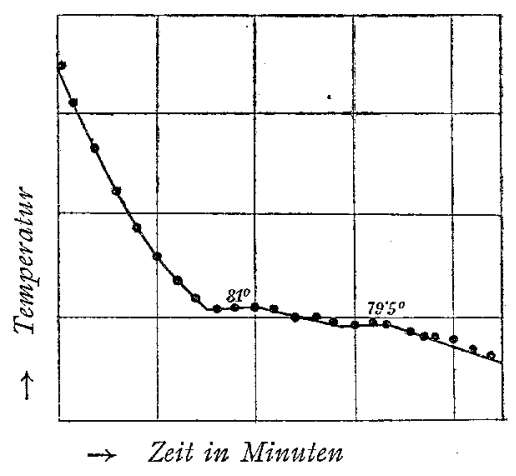

Fig. 7. bei $81.0^{\circ}$ die Verbindung aus; damit nähert sich die Zusammensetzung der Mutterlauge immer mehr dem eutektischen Punkte $a$, was mit dem Sinken der Temperatur von $81^{\circ}$ ab Hand in Hand geht. Ist schließlich die Zusammensetzung der eutektischen Mischung $a$ erreicht, so erstarrt diese homogen bei $79.5^{\circ}$, welche Temperatur die des eutektischen Punktes $a$ darstellt.

Lassen wir eine Mischung von 67 Molekülprozent Phenanthren erstarren, so erhalten wir bei der Temperaturbeobachtung jeder Minute während des Erstarrens der Reihe nach $108,105,101 \cdot 5,99,97 \cdot 5,95,92,90,87 \cdot 5,87,86,85 \cdot 0,83 \cdot 9,82 \cdot 8,82$, $81,80,79 \cdot 5,79 \cdot 5,79 \cdot 5,79,78 \cdot 5,78,77 \cdot 5,77,76 \cdot 5,76$.

Die Mischung obiger Zusammensetzung liegt bereits auf der Löslichkeitskurve $p$-Dinitrobenzol. Bei $87^{\circ}$ beginnt, wie aus der Zeit-Abkühlungskurve Fig. $8 \mathrm{zu}$ ersehen ist, die 
Ausscheidung von reinem Dinitrobenzol. Nun ändert sich unter steter langsamer Temperaturabnahme infolge der Ausscheidung von Dinitrotoluol die Zusammensetzung der Mutterlauge insolange, als die Zusammensetzung derselben der eutektischen Mischung a der Fig. 5 entspricht; dann wird alles fest, was dem erneuten Anhalten der Zeit-Abkühlungskurve (Fig. 8) bei $79 \cdot 5^{\circ}$ entspricht.

Wir kommen also zum Resultat, daß Phenanthren mit $p$-Dinitrobenzol zu einer Verbindung, bestehend aus 3 Molekülen Phenanthren und 1 Molekül Dinitrobenzol vom Schmelzpunkt 81 bis $81.5^{\circ}$ zusammentritt. Der eutektische Punkt

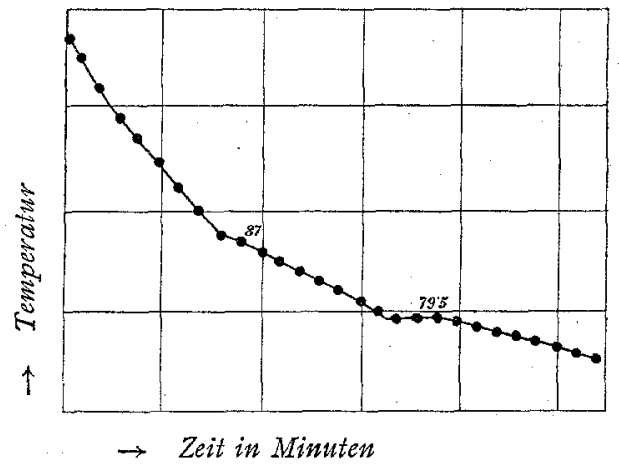

Fig. 8.

$z$ wischen der Verbindung und $p$-Dinitrobenzol liegt bei einer Zusammensetzung von 70 Molekülprozenten Phenanthren und 30 Molekülprozenten $p$-Dinitrobenzol und bei $79.5^{\circ}$ (Punkt $a$ der Fig. 5). Der eutektische Punkt zwischen der Verbindung und Phenanthren liegt bei einer Zusammensetzung von 77 Molekülprozenten Phenanthren und 23 Molekülprozenten $p$-Dinitrobenzol und bei $79^{\circ}$ (Punkt $b$ der Fig. 5).

Die beiden anderen Dinitrobenzole geben, wie eingangs erwähnt, mit Phenanthren keine Verbindungen.

4. Lösungsgleichgewichte zwischen Phenanthren und den isomeren Dinitrotoluolen $1,2,4 ; 1,2,6 ; 1,3,4$.

Die Versuchsergebnisse geben die folgenden Tabellen wieder. 
Tabelle 10 (Beobachter: K. Kaas und P. Dolch). Lösungsgleichgewicht zwischen Dinitrotoluol $(1,2,6)$ und Phenanthren.

a) Zusatz von Dinitrotoluol zu Phenanthren.

Menge Phenanthren: $8.252 \mathrm{~g}$.

\begin{tabular}{|c|c|c|c|}
\hline $\begin{array}{l}\text { Zusatz von } \\
\text { Dinitrotoluol }\end{array}$ & $\begin{array}{l}\text { Gewichtsprozente } \\
\text { Phenanthren }\end{array}$ & $\begin{array}{l}\text { Molekiulprozente } \\
\text { Phenanthren }\end{array}$ & Schmelzpunkt \\
\hline 0.000 & $100 \cdot 0$ & $100 \cdot 0$ & $103 \cdot 5$ \\
\hline $0 \cdot 426$ & $95 \cdot 1$ & $95 \cdot 2$ & $100 \cdot 5$ \\
\hline 0.818 & $91 \cdot 0$ & $91 \cdot 2$ & $97 \cdot 2$ \\
\hline $1 \cdot 526$ & 84.4 . & $84 \cdot 7$ & $92 \cdot 0$ \\
\hline $2 \cdot 276$ & $78 \cdot 4$ & $78 \cdot 7$ & $87 \cdot 5$ \\
\hline $3 \cdot 223$ & $71 \cdot 9$ & $72 \cdot 3$ & $81 \cdot 5$ \\
\hline $4 \cdot 317$ & $65 \cdot 7$ & $66 \cdot 2$ & $75 \cdot 5$ \\
\hline $5 \cdot 171$ & $61 \cdot 5$ & $62 \cdot 0$ & $72 \cdot 5$ \\
\hline $6 \cdot 159$ & $57 \cdot 3$ & $57 \cdot 8$ & $68 \cdot 0$ \\
\hline $7 \cdot 255$ & $53 \cdot 2$ & $53 \cdot 8$ & $63 \cdot 0$ \\
\hline $8 \cdot 625$ & $48 \cdot 9$ & $49 \cdot 4$ & $57 \cdot 5$ \\
\hline $9 \cdot 930$ & $45 \cdot 4$ & $\because \quad 46.0$ & $53 \cdot 0 \cdots$ \\
\hline $11 \cdot 365$ & $42 \cdot 1$ & $42 \cdot 6$ & $48 \cdot 0$ \\
\hline
\end{tabular}

b) Zusatz von Phenanthren zu Dinitrotoluol.

Menge Dinitrotoluol: $7 \cdot 540 \mathrm{~g}$.

\begin{tabular}{|c|c|c|c|}
\hline $\begin{array}{c}\text { Zusatz von } \\
\text { Phenanthren }\end{array}$ & $\begin{array}{c}\text { Gewichtsprozente } \\
\text { Phenanthren }\end{array}$ & $\begin{array}{c}\text { Molekiilprozente } \\
\text { Phenanthren }\end{array}$ & Schmelzpunkt \\
\hline \hline 0.000 & 0.0 & 0.0 & 65.0 \\
0.156 & 2.0 & $2 \cdot 1$ & 64.0 \\
0.669 & 8.2 & 8.3 & 60.5 \\
1.324 & 14.9 & 15.2 & 57.0
\end{tabular}


c) Zusatz von Phenanthren zu Dinitrotoluol.

Menge Dinitrotoluol: $5 \cdot 290 \mathrm{~g}$.

\begin{tabular}{|c|c|c|c|}
\hline $\begin{array}{c}\text { Zusatz von } \\
\text { Phenanthren }\end{array}$ & $\begin{array}{c}\text { Gewichtsprozente } \\
\text { Phenanthren }\end{array}$ & $\begin{array}{c}\text { Molekïlprozente } \\
\text { Phenanthren }\end{array}$ & Schmelzpunkt \\
\hline \hline $1 \cdot 32$ & $20 \cdot 0$ & $20 \cdot 3$ & $54 \cdot 0$ \\
$1 \cdot 61$ & $23 \cdot 3$ & $23 \cdot 7$ & $53 \cdot 0$ \\
$2 \cdot 01$ & $27 \cdot 5$ & $28 \cdot 0$ & $48 \cdot 0$ \\
$2 \cdot 40$ & $31 \cdot 2$ & $31 \cdot 7$ & $45 \cdot 0$ \\
$2 \cdot 97$ & $35 \cdot 9$ & $36 \cdot 5 \cdot 1$ & $40 \cdot 0$ \\
$3 \cdot 58$ & $40 \cdot 6$ & $41 \cdot 2$ & $44 \cdot 0$ \\
$4 \cdot 10$ & $43 \cdot 6$ & $44 \cdot 2$ & $49 \cdot 0$ \\
$4 \cdot 47$ & $45 \cdot 9$ & $46 \cdot 5$ & $52 \cdot 0$ \\
& & &
\end{tabular}

Tabelle 11 (Beobachter: K. Kaas).

Lösungsgleichgewicht $z$ wischen Dinitrotoluol $(1,2,4)$ und Phenanthren.

a) Zusatz von Phenanthren zu Dinitrotoluol.

Menge Dinitrotoluo1: $8 \cdot 900 \mathrm{~g}$.

\begin{tabular}{|c|c|c|c|}
\hline $\begin{array}{c}\text { Zusatz von } \\
\text { Phenanthren }\end{array}$ & $\begin{array}{c}\text { Gewichtsprozente } \\
\text { Phenanthren }\end{array}$ & $\begin{array}{c}\text { Molekiulprozente } \\
\text { Phenanthren }\end{array}$ & Schmelzpunkt \\
\hline \hline $0 \cdot 000$ & $0 \cdot 0$ & $0 \cdot 0$ & $69 \cdot 0$ \\
$0 \cdot 291$ & $3 \cdot 2$ & $3 \cdot 2$ & $68 \cdot 0$ \\
$0 \cdot 714$ & $7 \cdot 4$ & $7 \cdot 6$ & $65 \cdot 5$ \\
$1 \cdot 091$ & $10 \cdot 9$ & $11 \cdot 1$ & $64 \cdot 0$ \\
$1 \cdot 489$ & $14 \cdot 3$ & $14 \cdot 6$ & $61 \cdot 5$ \\
$1 \cdot 927$ & $17 \cdot 8$ & $18 \cdot 1$ & $60 \cdot 0$ \\
$2 \cdot 301$ & $20 \cdot 6$ & $20 \cdot 9$ & $58 \cdot 0$ \\
$2 \cdot 818$ & $24 \cdot 0$ & $24 \cdot 4$ & $56 \cdot 0$ \\
$3 \cdot 169$ & $26 \cdot 3$ & $26 \cdot 7$ & $54 \cdot 5$ \\
$3 \cdot 460$ & $27 \cdot 9$ & $18 \cdot 4$ & $53 \cdot 0$ \\
$3 \cdot 855$ & $30 \cdot 2$ & $30 \cdot 7$ & $51 \cdot 0$ \\
$4 \cdot 512$ & $33 \cdot 7$ & $34 \cdot 2$ & $49 \cdot 0$ \\
$5 \cdot 040$ & $36 \cdot 2$ & $36 \cdot 2$ & $46 \cdot 7$ \\
$5 \cdot 565$ & $38 \cdot 5$ & $39 \cdot 0$ & $44 \cdot 0$ \\
$6 \cdot 306$ & $41 \cdot 5$ & $42 \cdot 0$ & $41 \cdot 0$ \\
$7 \cdot 284$ & $45 \cdot 0$ & $45 \cdot 6$ & $30 \cdot 0$ \\
$8 \cdot 341$ & $48 \cdot 4$ & $48 \cdot 9$ & $44 \cdot 5$ \\
& & &
\end{tabular}


b) Zusatz von Dinitrotoluol zu Phenanthren.

Menge Phenanthren: $8 \cdot 095 \mathrm{~g}$.

\begin{tabular}{|c|c|c|c|}
\hline $\begin{array}{c}\text { Zusatz von } \\
\text { Phenanthren }\end{array}$ & $\begin{array}{c}\text { Gewichtsprozente } \\
\text { Phenanthren }\end{array}$ & $\begin{array}{c}\text { Molekuilprozente } \\
\text { Phenanthren }\end{array}$ & Schmelzpunkt \\
\hline \hline $0 \cdot 000$ & $100 \cdot 0$ & $100 \cdot 0$ & $103 \cdot 5$ \\
$0 \cdot 680$ & $92 \cdot 2$ & $92 \cdot 4$ & $98 \cdot 0$ \\
$1 \cdot 152$ & $87 \cdot 5$ & $87 \cdot 8$ & $95 \cdot 0$ \\
$1 \cdot 602$ & $84 \cdot 6$ & $84 \cdot 9$ & $91 \cdot 7$ \\
$2 \cdot 204$ & $78 \cdot 6$ & $79 \cdot 0$ & $87 \cdot 0$ \\
$2 \cdot 936$ & $73 \cdot 4$ & $73 \cdot 8$ & $81 \cdot 5$ \\
$3 \cdot 436$ & $70 \cdot 2$ & $70 \cdot 7$ & $78 \cdot 0$ \\
$4 \cdot 159$ & $61 \cdot 1$ & $66 \cdot 6$ & $73 \cdot 5$ \\
$4 \cdot 902$ & $62 \cdot 3$ & $62 \cdot 8$ & $68 \cdot 0$ \\
$5 \cdot 559$ & $59 \cdot 3$ & $59 \cdot 8$ & $63 \cdot 0$ \\
$6 \cdot 159$ & $56 \cdot 8$ & $57 \cdot 3$ & $59 \cdot 5$ \\
$7 \cdot 359$ & $52 \cdot 4$ & $52 \cdot 9$ & $53 \cdot 0$ \\
$8 \cdot 541$ & $48 \cdot 6$ & $49 \cdot 2$ & $46 \cdot 0$ \\
$9 \cdot 424$ & $46 \cdot 2$ & $46 \cdot 8$ & $42 \cdot 0$ \\
$10 \cdot 332$ & $43 \cdot 9$ & $44 \cdot 3$ & $37 \cdot 5$ \\
& & & \\
\hline
\end{tabular}

Tabelle 12 (Beobachter: F. Scherenziss).

Lösungsgleichgewicht zwischen Dinitrotoluol $(1,3,4)$ und Phenanthren.

a) Zusatz von Dinitrotoluol zu Phenanthren.

Menge Phenanthren: $8 \cdot 245 \mathrm{~g}$.

\begin{tabular}{|c|c|c|c|}
\hline $\begin{array}{l}\text { Zusatz von } \\
\text { Dinitrotoluol }\end{array}$ & $\begin{array}{c}\text { Gewichtsprozente } \\
\text { Phenanthren }\end{array}$ & $\begin{array}{l}\text { Molekülprozente } \\
\text { Phenanthren }\end{array}$ & Schmelzpunkt \\
\hline 0.000 & $100 \cdot 0$ & $100 \cdot 0$ & $103 \cdot 5$ \\
\hline 0.543 & $93 \cdot 8$ & 94.0 & $99 \cdot 5$ \\
\hline $1 \cdot 037$ & $88 \cdot 8$ & $89 \cdot 1$ & $96 \cdot 5$ \\
\hline $1 \cdot 595$ & $83 \cdot 8$ & $84 \cdot 1$ & $92 \cdot 2$ \\
\hline $2 \cdot 311$ & $78 \cdot 2$ & $78 \cdot 6$ & $87 \cdot 5$ \\
\hline $3 \cdot 300$ & $71 \cdot 4$ & $71 \cdot 8$ & $82 \cdot 0$ \\
\hline $4 \cdot 230$ & $66 \cdot 1$ & $66 \cdot 6$ & $77 \cdot 0$ \\
\hline $5 \cdot 580$ & $58 \cdot 9$ & $59 \cdot 4$ & $69 \cdot 0$ \\
\hline $7 \cdot 170$ & $53 \cdot 8$ & $54 \cdot 3$ & $61 \cdot 0$ \\
\hline $8 \cdot 563$ & $49 \cdot 0$ & $49 \cdot 6$ & $55 \cdot 0$ \\
\hline
\end{tabular}


b) Zusatz von Phenanthren zu Dinitrotoluol.

Menge Dinitrotoluol: $8 \cdot 262 \mathrm{~g}$.

\begin{tabular}{|c|c|c|c|}
\hline $\begin{array}{c}\text { Zusatz von } \\
\text { Phenanthren }\end{array}$ & $\begin{array}{c}\text { Gewichtsprozente } \\
\text { Phenanthren }\end{array}$ & $\begin{array}{c}\text { Molekülprozente } \\
\text { Phenanthren }\end{array}$ & Schmelzpunkt \\
\hline \hline 0.000 & $0 \cdot 0$ & $0 \cdot 0$ & $59 \cdot 0$ \\
1.159 & $12 \cdot 3$ & $12 \cdot 5$ & $51 \cdot 5$ \\
1.384 & $14 \cdot 4$ & $14 \cdot 7$ & $49 \cdot 0$ \\
1.654 & $16 \cdot 7$ & $17 \cdot 0$ & $47 \cdot 0$ \\
$2 \cdot 206$ & $21 \cdot 1$ & $21 \cdot 5$ & $44 \cdot 0$ \\
$3 \cdot 074$ & $27 \cdot 2$ & $27 \cdot 6$ & $40 \cdot 0$ \\
$3 \cdot 827$ & $31 \cdot 6$ & $32 \cdot 1$ & $36 \cdot 0$ \\
$4 \cdot 219$ & $32 \cdot 3$ & $32 \cdot 8$ & $34 \cdot 0$ \\
5.854 & $41 \cdot 4$ & $41 \cdot 9$ & $42 \cdot 8$ \\
$7 \cdot 035$ & $44 \cdot 7$ & $45 \cdot 3$ & $50 \cdot 9$ \\
8.328 & $50 \cdot 5$ & $51 \cdot 1$ & $57 \cdot 6$ \\
& & &
\end{tabular}

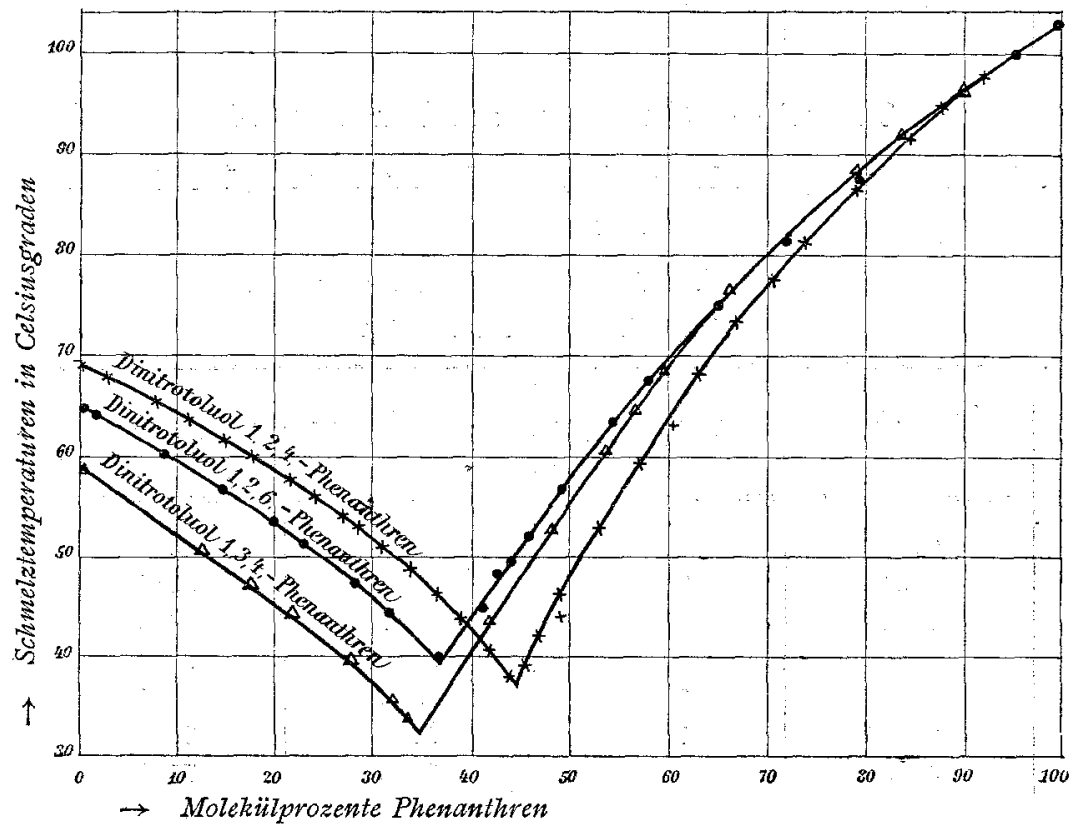

Fig. 9.

Lösungsgleichgewicht zwischen Dinitrotoluolen und Phenanthren. 
Die graphische Darstellung in Fig. 9 stellt die Versuchsdaten anschaulich dar.

Ich kann mich bei der Diskussion der Versuchsergebnisse ganz kurz fassen. Aus dem Verlauf der Schmelzkurven geht eindeutig hervor, daß keines der drei untersuchten Dinitrotoluole mit Phenanthren zu einer Verbindung zusammentritt. Die Lage und Zusammensetzung der eutektischen Punkte ergibt sich ohneweiters aus den Schmelzkurven der Fig. 9.

\section{Lösungsgleichgewichte zwischen Phenanthren und Trinitro- benzol, beziehungsweise Trinitrotoluol.}

Die Versuchsergebnisse geben die beiden folgenden Tabellen 13 und 14 wieder.

Tabelle 13 (Beobachter: P. Dolch).

Lösungsgleichgewicht zwischen Phenanthren und Trinitrotoluol.

a) Zusatz von Trinitrotoluol zu Phenanthren.

Menge Phenanthren: $7 \cdot 68 \mathrm{~g}$.

\begin{tabular}{|c|c|c|c|}
\hline $\begin{array}{c}\text { Zusatz von } \\
\text { Trinitrotoluol }\end{array}$ & $\begin{array}{c}\text { Gewichtsprozente } \\
\text { Phenanthren }\end{array}$ & $\begin{array}{c}\text { Molekülprozente } \\
\text { Phenanthren }\end{array}$ & Schmelzpunkt \\
\hline \hline 0.00 & $100 \cdot 0$ & $100 \cdot 0$ & $103 \cdot 0$ \\
$0 \cdot 42$ & $94 \cdot 8$ & $95 \cdot 9$ & $101 \cdot 5$ \\
$0 \cdot 77$ & $90 \cdot 9$ & $92 \cdot 8$ & $98 \cdot 5$ \\
1.13 & $87 \cdot 2$ & $89 \cdot 8$ & $96 \cdot 0$ \\
1.87 & $80 \cdot 4$ & $84 \cdot 1$ & $92 \cdot 0$ \\
$2 \cdot 49$ & $75 \cdot 5$ & $79 \cdot 6$ & $87 \cdot 0$ \\
$4 \cdot 06$ & $65 \cdot 4$ & $70 \cdot 7$ & $77 \cdot 0$ \\
$4 \cdot 62$ & $62 \cdot 4$ & $67 \cdot 8$ & $77 \cdot 5$ \\
$5 \cdot 10$ & $60 \cdot 1$ & 65.8 & $79 \cdot 0$ \\
$5 \cdot 78$ & $57 \cdot 1$ & $62 \cdot 9$ & $82 \cdot 5$ \\
$6 \cdot 67$ & $53 \cdot 4$ & $59 \cdot 4$ & $85 \cdot 0$ \\
$7 \cdot 82$ & $49 \cdot 6$ & $55 \cdot 6$ & $86 \cdot 5$ \\
$8 \cdot 29$ & $48 \cdot 1$ & $54 \cdot 1$ & $87 \cdot 0$ \\
$9 \cdot 18$ & $45 \cdot 6$ & $51 \cdot 7$ & $87 \cdot 5$ \\
& & & \\
\hline
\end{tabular}


b) Zusatz von Phenanthren zu Trinitrotoluol.

Menge Trinitrotoluol: $6.97 \mathrm{~g}$.

\begin{tabular}{|c|c|c|c|}
\hline $\begin{array}{l}\text { Zusatz von } \\
\text { Phenanthren }\end{array}$ & $\begin{array}{l}\text { Gewichtsprozente } \\
\text { Phenanthren }\end{array}$ & $\begin{array}{c}\text { Molekülprozente } \\
\text { Phenanthren }\end{array}$ & Schmelzpunkt \\
\hline 0.00 & 0.00 & 0.0 & $78 \cdot 0$ \\
\hline 0.63 & $8 \cdot 3$ & $10 \cdot 4$ & $74 \cdot 0$ \\
\hline $1 \cdot 14$ & $14 \cdot 0$ & $17 \cdot 2$ & $70 \cdot 0$ \\
\hline $1 \cdot 48$ & $17 \cdot 4$ & $21 \cdot 3$ & $72 \cdot 0$ \\
\hline $2 \cdot 24$ & $24 \cdot 3$ & $29 \cdot 1$ & $78 \cdot 5$ \\
\hline $2 \cdot 70$ & $27 \cdot 9$ & $33 \cdot 1$ & $82 \cdot 0$ \\
\hline $3 \cdot 48$ & $33 \cdot 4$ & $39 \cdot 0$ & $85 \cdot 0$ \\
\hline $4 \cdot 49$ & $39 \cdot 0$ & $44 \cdot 9$ & $87 \cdot 0$ \\
\hline $4 \cdot 80$ & $43 \cdot 4$ & $49 \cdot 5$ & $87 \cdot 5$ \\
\hline
\end{tabular}

Tabelle 14 (Beobachter: P. Dolch).

Lösungsgleichgewicht $z$ wischen Trinitrobenzol und Phenanthren.

a) Zusatz von Trinitrobenzol zu Phenanthren.

Menge Phenanthren: $5 \cdot 11 \mathrm{~g}$.

\begin{tabular}{|c|c|c|c|}
\hline $\begin{array}{l}\text { Zusatz von } \\
\text { Trinitrobenzol }\end{array}$ & $\begin{array}{l}\text { Gewichtsprozente } \\
\text { Phenanthren }\end{array}$ & $\begin{array}{l}\text { Molekülprozente } \\
\text { Phenanthren }\end{array}$ & Schmelzpunkt \\
\hline 0.00 & $100 \cdot 0$ & $100 \cdot 0$ & 103 \\
\hline 0.37 & $93 \cdot 2$ & $94 \cdot 2$ & 100 \\
\hline 0.57 & $89 \cdot 9$ & $91 \cdot 5$ & 97 \\
\hline 0.85 & $80 \cdot 8$ & $87 \cdot 8$ & 94 \\
\hline 1.04 & $83 \cdot 1$ & $85 \cdot 6$ & 91 \\
\hline $1 \cdot 26$ & $80 \cdot 2$ & $82 \cdot 6$ & 91 \\
\hline $1 \cdot 77$ & $75 \cdot 5$ & $78 \cdot 7$ & 102 \\
\hline $2 \cdot 28$ & $69 \cdot 1$ & $72 \cdot 8$ & 111 \\
\hline $2 \cdot 84$ & $64 \cdot 3$ & $6 \dot{8} \cdot 2$ & 117 \\
\hline $3 \cdot 36$ & $60 \cdot 3$ & $64 \cdot 5$ & 120 \\
\hline $4 \cdot 41$ & $53 \cdot 7$ & $58 \cdot 1$ & 124 \\
\hline $5 \cdot 42$ & $48 \cdot 5$ & $52 \cdot 9$ & 125 \\
\hline
\end{tabular}


Einfluß von Substitution etc.

b) Zusatz von Phenanthren zu Trinitrobenzol.

Menge Trinitrobenzol: $5 \cdot 67 \mathrm{~g}$.

\begin{tabular}{|c|c|c|c|}
\hline $\begin{array}{c}\text { Zusatz von } \\
\text { Phenanthren }\end{array}$ & $\begin{array}{c}\text { Gewichtsprozente } \\
\text { Phenanthren }\end{array}$ & $\begin{array}{c}\text { Molekülprozente } \\
\text { Phenanthren }\end{array}$ & Schmelzpunkt \\
\hline & 0.0 & 0.0 & 121 \\
0.63 & 10.0 & 11.7 & 111 \\
1.07 & 15.9 & 18.5 & 106 \\
1.85 & 24.6 & $27 \cdot 1$ & 115 \\
3.47 & 38.0 & 42.1 & 124 \\
& & & \\
\hline
\end{tabular}

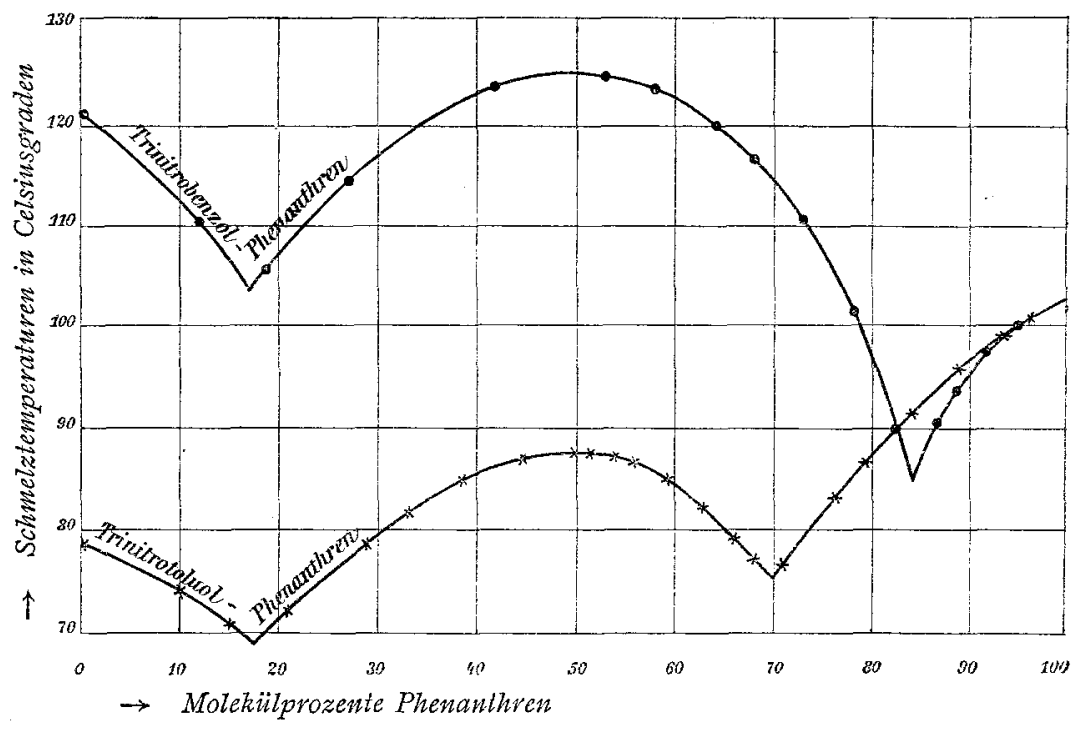

Fig. 10.

Lösungsgleichgewichte zwischen Trinitrobenzol und -toluol und Phenanthren.

In Fig. 10 sind die experimentellen Daten graphisch veranschaulicht. Man sieht, daß Phenanthren sowohl mit Trinitrobenzol als auch mit Trinitrotoluol zu Verbindungen im äquimolekularen Verhältnis zusammentreten, die Schmelzpunkte von 125 , beziehungsweise $87 \cdot 5^{\circ}$ aufweisen. Wir haben in beiden Fällen daher zwei eutektische Punkte.

Der eutektische Punkt zwischen Trinitrobenzol und der Verbindung liegt bei 17 Molekülprozenten Phenanthren und 
bei $104^{\circ}$, der $z$ wischen der Verbindung und Phenanthren liegt bei einer Zusammensetzung von $84 \cdot 5$ Molekülprozenten Phenanthren und $85.5^{\circ}$.

Zwischen 17 und 84.5 Molekülprozenten Phenanthren liegt das Existenzbereich der Verbindung Phenanthren-Trinitrobenzol.

Das Existenzbereich der Verbindung Trinitrotoluol-Phenanthren liegt zwischen Zusammensetzung der Mischung von 18 bis 70 Molekülprozenten Phenanthren. Dies sind auch die Zusammensetzungen der eutektischen Punkte von Trinitrotoluol und der Verbindung einerseits, der Verbindung und Phenanthren andrerseits. Die entsprechenden Temperaturwerte der beiden eutektischen Punkte liegen bei $69^{\circ}$, beziehungsweise $70^{\circ}$. 